\title{
On approximation of the continuous functions of two variables by the Fourier-Haar "angle"
}

\author{
Alexander N. Shchitov \\ Dnipropetrovsk, Ukraine \\ an_shchitov@rambler.ru
}

Keywords: Fourier-Haar system, Fourier-Haar sums, Fourier-Haar "angle", exact constant, function of two variables, modulus of continuity, approximation, uniform metric.

\begin{abstract}
The exact value of the upper bound of the approximation error by the construction of the type "angle" built using the Fourier-Haar partial sums is obtained on the one function class of two variables in the uniform metric.
\end{abstract}

\section{Introduction}

Haar system was introduced in 1909 [1]. This system of functions is orthonormal on [0,1]. The properties of the Haar system as well as the behavior of the Fourier-Haar coefficients were studied by P.L. Ulyanov [2], Z. Ciesielski [3], B.I. Golubov [4], I.I. Sharapudinov [5], S.Yu. Galkina [6,7], S.S. Volosivets [8], A.N. Shchitov [9, 10], M.G. Plotnikov [11] and a lot of other scientists. Some of the obtained results can be found in the review article [12].

In approximation theory a lot of works are devoted to the solving problems of the approximation of functions of one and several variables by polynomials in the Haar system and partial Fourier-Haar sums.

For example, in the articles N.P. Khoroshko $[13,14]$ in the uniform and integral metrics were obtained the exact estimates of the approximation errors of the classes of the continuity functions of one variable by polynomials in the Haar system and partial Fourier-Haar sums. In the articles N.P. Khoroshko [13], S.B. Vakarchuk and A.N. Schitov [15] were obtained the exact estimates of the best approximation by the polynomials in the Haar system of the functions of boundary variation in the integral metric. The exact estimates of the errors of the sided approximation by the polynomials in the Haar system in uniform and integral metrics were obtained for some function classes by S.B. Vakarchuk and A.N. Schitov in the article [16]. In the case of the classes of the differentiable functions of one variable the exact estimates of the approximation errors by the polynomials in the Haar system were obtained in the metrics $L_{p}(1 \leqslant p \leqslant \infty)$ in the article S.B. Vakarchuk and A.N. Schitov [17].

The questions of obtaining of the upper bounds of the approximation of the continuity functions of two and more variables by polynomials in the Haar system using the modulus of continuity were considered by A.R. Abdulgamidov [18], N.D. Rishchenko [19], M.N. Ochirov [20] and L.G. Chomutenko [21]. P.V. Zaderey and N.N. Zaderey [22], S.B. Vakarchuk and A.N. Schitov [23] have obtained the exact estimates of the approximation errors of the classes of the continuity functions of several variables by polynomials in the Haar system and partial Fourier-Haar sums in the uniform metric.

In this paper we continue to study the approximation properties of the Haar system. We consider the approximation of the continuity functions of two variables by the construction of the type "angle" $\sigma_{n_{1}, n_{2}}\left(f ; t_{1}, t_{2}\right)$ that built using the Fourier-Haar partial sums.

We can note that in the case of the trigonometric polynomials approximations by the constuctions of the type "angle" were considered by many mathematicians. For example, M.N. Potapov [24, 25] obtained several direct theorem of the approximation theory using the mixed modulus of continuity. 


\section{Theory}

Let $\mathbb{I}^{2}=\left\{\mathbf{t}=\left(t_{1}, t_{2}\right): 0 \leqslant t_{i} \leqslant 1, i=\overline{1,2}\right\}$ - 2-dimensional cube in the space $\mathbb{R}^{2}$. On the unit segment $\mathbb{I}=[0,1]$ we consider the binary intervals that is defined in the following way: let for arbitrary number $n_{i}=2^{m_{i}}+k_{i}\left(m_{i} \in \mathbb{Z}_{+}, k_{i}=\overline{1,2^{m_{i}}}\right)$

$$
\delta_{n_{i}} \equiv \delta_{m_{i}}^{k_{i}}=\left(\left(k_{i}-1\right) / 2^{m_{i}}, k_{i} / 2^{m_{i}}\right) .
$$

On the $[0,1]$ we define the Haar system [1]: $\chi_{1}(t) \equiv \chi_{0}^{(0)}(t) \equiv 1$, and for $n_{i}=2^{m_{i}}+k_{i}\left(m_{i} \in \mathbb{Z}_{+}, k_{i}=\right.$ $\left.\overline{1,2^{m_{i}}}\right)$

$$
\chi_{n_{i}}(t) \equiv \chi_{m_{i}}^{\left(k_{i}\right)}(t)=\left\{\begin{array}{cl}
2^{m_{i} / 2} & , \text { if } t \in \delta_{m_{i}+1}^{2 k_{i}-1}, \\
-2^{m_{i} / 2} & , \text { if } t \in \delta_{m_{i}}^{2 k_{i}}, \\
0 & , \text { if } t \bar{\in} \overline{\delta_{m_{i}}^{k_{i}}},
\end{array}\right.
$$

where $\overline{\delta_{m_{i}}^{k_{i}}}$ - the closure of the set $\delta_{m_{i}}^{k_{i}}$. At the points of discontinuity the Haar functions are equal to the average of the left and right limits of the Haar functions. At the ends of the segment $[0,1]$ the Haar functions are equal to the limit values from the inside of the segment. Let $\mathbb{N}^{2} \stackrel{\text { df }}{=}\left\{\mathbf{n}=\left(n_{1}, n_{2}\right)\right.$ : $\left.n_{i} \in \mathbb{N}, i=\overline{1,2}\right\}, \mathbb{N}_{*}^{2} \stackrel{\text { df }}{=}\left\{\mathbf{n}=\left(n_{1}, n_{2}\right): n_{i} \in \mathbb{N} \backslash\{1\}, i=\overline{1,2}\right\}$. The set $\left\{\chi_{\mathbf{n}}(\mathbf{t}) \stackrel{\text { df }}{=} \prod_{i=1}^{2} \chi_{n_{i}}\left(t_{i}\right)\right\}_{\mathbf{n} \in \mathbb{N}^{2}}$ forms the orthonormal system of Haar functions on the 2-dimensional cube $\mathbb{I}^{2}$. Information about Haar system can be found, for example, in the books [26-29].

Let $\mathbf{c}_{\mathbf{n}}(f) \equiv \mathbf{c}_{\mathbf{m}}^{\mathbf{k}}(f)=\int_{\mathbb{T}^{2}} f(\mathbf{t}) \chi_{\mathbf{n}}(\mathbf{t}) \mathbf{d t}\left(\mathbf{n} \in \mathbb{N}^{2}\right)$, where $\mathbf{d t} \stackrel{\mathrm{d} f}{=} \prod_{i=1}^{2} \mathrm{~d} t_{i}$, are the Fourier-Haar coefficients on the unit cube $\mathbb{I}^{2}$ of the function $f(\mathbf{t})$. Let

$$
S_{\mathbf{n}}(f ; \mathbf{t})=\sum_{\substack{\mathbf{k} \in \mathbb{N}^{2} \\\left(k_{i} \leqslant n_{i}, i=\overline{1,2}\right)}} \mathbf{c}_{\mathbf{k}}(f) \chi_{\mathbf{k}}(\mathbf{t})=\int_{\mathbb{I}^{2}} f(\mathbf{x}) K_{\mathbf{n}}(\mathbf{x} ; \mathbf{t}) \mathbf{d} \mathbf{x}
$$

is the partial rectangular sum of the Fourier-Haar series, where

$$
K_{\mathbf{n}}(\mathbf{x} ; \mathbf{t}) \stackrel{\mathrm{df}}{=} \prod_{i=1}^{2} K_{n_{i}}\left(x_{i} ; t_{i}\right), \quad K_{n_{i}}\left(x_{i} ; t_{i}\right) \stackrel{\mathrm{df}}{=} \sum_{j=1}^{n_{i}} \chi_{j}\left(x_{i}\right) \chi_{j}\left(t_{i}\right) .
$$

Let $h_{i} \stackrel{\mathrm{df}}{=} 2^{-\left(m_{i}+1\right)}$ and

$$
n_{i}^{\prime} \stackrel{\text { df }}{=} \begin{cases}2^{m_{i}} & , \text { if } n_{i}=2^{m_{i}}+k_{i}\left(m_{i} \in \mathbb{N} ; k_{i}=\overline{1,2^{m_{i}}-1}\right) \\ 2^{m_{i}+1} & , \text { if } n_{i}=2^{m_{i}+1}\left(m_{i} \in \mathbb{Z}_{+}\right) ; i=\overline{1,2}\end{cases}
$$

For $n_{i}=2^{m_{i}}+k_{i}\left(m_{i} \in \mathbb{Z}_{+} ; k_{i}=\overline{1,2^{m_{i}}}\right)$ we denote

$$
\Lambda_{m_{i}}^{\nu_{i}} \stackrel{\text { df }}{=} \begin{cases}\delta_{m_{i}+1}^{\nu_{i}} & , \text { if } 1 \leqslant \nu_{i} \leqslant 2 k_{i}, \\ \delta_{m_{i}}^{\eta_{i}} & , \text { if } 2 k_{i}+1 \leqslant \nu_{i} \leqslant n_{i}, \eta_{i}=\nu_{i}-k_{i} .\end{cases}
$$

Let $C \equiv C\left(\mathbb{I}^{2}\right)$ is the line space of the continuity on the $\mathbb{I}^{2}$ functions $f(\mathbf{t})$ with the norm $\|f\|_{C} \stackrel{\text { df }}{=}$ $\max \left\{|f(\mathbf{t})|: \mathbf{t} \in \mathbb{I}^{2}\right\}$. 
Let

$$
\left\{\begin{array}{l}
S_{n_{1}, \infty}\left(f ; t_{1}, t_{2}\right) \stackrel{\mathrm{df}}{=} \int_{0}^{1} f\left(u, t_{2}\right) K_{n_{1}}\left(u ; t_{1}\right) \mathrm{d} u, \\
S_{\infty, n_{2}}\left(f ; t_{1}, t_{2}\right) \stackrel{\mathrm{df}}{=} \int_{0}^{1} f\left(t_{1}, v\right) K_{n_{2}}\left(v ; t_{2}\right) \mathrm{d} v
\end{array}\right.
$$

are the partial sums of the Fourier-Haar series obtained under the action of the operators $S_{n_{1}}(\cdot)$ and $S_{n_{2}}(\cdot)$ on the $f\left(t_{1}, t_{2}\right) \in C$ correspondingly as on the function of the $t_{1}$ (with fixed $t_{2}$ ) and on the function of the $t_{2}$ (with fixed $t_{1}$ ). From [30] we can write

$$
S_{n_{1}, n_{2}}(f)=S_{n_{1}, \infty}\left[S_{\infty, n_{2}}(f)\right]=S_{\infty, n_{2}}\left[S_{n_{1}, \infty}(f)\right]
$$

By using of the definitions of the Fourier-Haar partial sums, we build the two-dimensional "angle"

$$
\sigma_{n_{1}, n_{2}}\left(f ; t_{1}, t_{2}\right)=S_{n_{1}, \infty}\left(f ; t_{1}, t_{2}\right)+S_{\infty, n_{2}}\left(f ; t_{1}, t_{2}\right)-S_{n_{1}, n_{2}}\left(f ; t_{1}, t_{2}\right),
$$

(explanation of the term "angle" can be found, for example, in [25]).

By the mixed modulus of continuity of the function $f(\mathbf{t}) \in C$ we call the value

$$
\Omega(f ; \mathbf{u})=\sup \left\{\left|\triangle_{\mathbf{h}}^{\mathbf{1}} f(\mathbf{t})\right|:\left|h_{i}\right| \leqslant u_{i} ; t_{i}, t_{i}+h_{i} \in[0,1], i=\overline{1,2}\right\}\left(\mathbf{u} \in \mathbb{I}^{2}\right),
$$

where $\triangle_{\mathbf{h}}^{\mathbf{1}} f(\mathbf{t}) \stackrel{\mathrm{df}}{=} \triangle_{h_{1}}^{1} \triangle_{h_{2}}^{1} f(\mathbf{t}) ; \triangle_{h_{1}}^{1} f(\mathbf{t})=f\left(t_{1}+h_{1}, t_{2}\right)-f\left(t_{1}, t_{2}\right), \triangle_{h_{2}}^{1} f(\mathbf{t})=f\left(t_{1}, t_{2}+h_{2}\right)-$ $f\left(t_{1}, t_{2}\right)$ are the first differences of the function $f$ with the steps $h_{1}$ and $h_{2}$ on the variables $t_{1}$ and $t_{2}$ accordingly.

We set the next function class

$$
H_{\Omega} \stackrel{\text { df }}{=}\left\{f(\mathbf{t}) \in C: \Omega(f ; \mathbf{u}) \leqslant \Omega(\mathbf{u}) \forall \mathbf{u} \in \mathbb{I}^{2}\right\}
$$

where $\Omega(\mathbf{u})\left(\mathbf{u} \in \mathbb{I}^{2}\right)$ is some defined modulus of continuity. The function $\Omega(\mathbf{u})$ is continuity, nonnegative and nondecreasing for each variables in the definition set of the function and such that $\Omega(\mathbf{u})=0$, if $\prod_{i=1}^{2} u_{i}=0$.

\section{Results}

In the next theorem is obtained the exact value of the approximation error of the continuity function of two variable from the function class $H_{\Omega}$ by the "angle" built with Fourier-Haar partial sums.

Theorem 1. For arbitrary modulus of continuity $\Omega(\mathbf{t})\left(\mathbf{t} \in \mathbb{I}^{2}\right)$ and for all numbers $n_{i}=2^{m_{i}}+k_{i}\left(m_{i} \in\right.$ $\left.\mathbb{Z}_{+} ; k_{i}=\overline{1,2^{m_{i}}} ; i=1,2\right)$ it is hold the equality

$$
\sup _{f \in H_{\Omega}}\left\|f-\sigma_{n_{1}, n_{2}}(f)\right\|_{C}=n_{1}^{\prime} n_{2}^{\prime} \int_{0}^{1 / n_{1}^{\prime}} \int_{0}^{1 / n_{2}^{\prime}} \Omega\left(t_{1}, t_{2}\right) \mathrm{d} t_{1} \mathrm{~d} t_{2} .
$$

We need the following two lemmas from the [23] to prove the theorem 1.

Lemma 1. Let on the set $\mathbb{R}_{+}^{2}$ is defined the continuous, nonnegative, nondecreasing on each variables function $f\left(t_{1}, t_{2}\right)$. Then the following equality holds 


$$
\max _{\left(t_{1}, t_{2}\right) \in\left[a_{1}, b_{1}\right] \times\left[a_{2}, b_{2}\right]} \int_{a_{1}}^{b_{1}} \int_{a_{2}}^{b_{2}} f\left(\left|t_{1}-u_{1}\right|,\left|t_{2}-u_{2}\right|\right) \mathrm{d} u_{1} \mathrm{~d} u_{2}=\int_{0}^{b_{1}-a_{1}} \int_{0}^{b_{2}-a_{2}} f\left(u_{1}, u_{2}\right) \mathrm{d} u_{1} \mathrm{~d} u_{2},
$$

where $0 \leqslant a_{i}<b_{i}<\infty(i=1,2)$.

Proof of the lemma 1. Let

$$
G\left(t_{1}, t_{2}\right) \stackrel{\mathrm{df}}{=} \int_{a_{1}}^{b_{1}} \int_{a_{2}}^{b_{2}} f\left(\left|t_{1}-u_{1}\right|,\left|t_{2}-u_{2}\right|\right) \mathrm{d} u_{1} \mathrm{~d} u_{2}
$$

We can write the equivalent form of this function:

$$
\begin{aligned}
G\left(t_{1}, t_{2}\right) & =\int_{0}^{t_{1}-a_{1}} \int_{0}^{t_{2}-a_{2}} f\left(u_{1}, u_{2}\right) \mathrm{d} u_{1} \mathrm{~d} u_{2}+\int_{0}^{t_{1}-a_{1}} \int_{0}^{t_{1}-t_{2}} f\left(u_{1}, u_{2}\right) \mathrm{d} u_{1} \mathrm{~d} u_{2}+ \\
& +\int_{0}^{t_{1}-a_{2}} \int_{0}^{b_{1}-t_{1}} f\left(u_{1}, u_{2}\right) \mathrm{d} u_{1} \mathrm{~d} u_{2}+\int_{0}^{b_{2}} \int_{0}^{t_{2}} f\left(u_{1}, u_{2}\right) \mathrm{d} u_{1} \mathrm{~d} u_{2} .
\end{aligned}
$$

After obtaining the first partial derivative on the variable $t_{1}$, we can write

$$
\begin{aligned}
G^{(1,0)}\left(t_{1}, t_{2}\right) & =\int_{0}^{t_{2}-a_{2}}\left[f\left(t_{1}-a_{1}, u_{2}\right)-f\left(b_{1}-t_{1}, u_{2}\right)\right] \mathrm{d} u_{2}+ \\
& +\int_{0}^{b_{2}-t_{2}}\left[f\left(t_{1}-a_{1}, u_{2}\right)-f\left(b_{1}-t_{1}, u_{2}\right)\right] \mathrm{d} u_{2} .
\end{aligned}
$$

On the segment $a_{1} \leqslant t_{1} \leqslant b_{1}$ we consider the auxiliary function

$$
g\left(t_{1}, u_{2}\right) \stackrel{\mathrm{df}}{=} f\left(t_{1}-a_{1}, u_{2}\right)-f\left(b_{1}-t_{1}, u_{2}\right) .
$$

For $a_{1} \leqslant t_{1}<\left(a_{1}+b_{1}\right) / 2$ and arbitrary $u_{2} \geqslant 0$ by the conditions of the lemma 1 we have $g\left(t_{1}, u_{2}\right) \leqslant 0$. Then from (9) $G^{(1,0)}\left(t_{1}, t_{2}\right) \leqslant 0$ for arbitrary point $\left(t_{1}, t_{2}\right) \in\left[a_{1},\left(a_{1}+b_{1}\right) / 2\right) \times\left[a_{2}, b_{2}\right]$. In the case $\left(a_{1}+b_{1}\right) / 2<t_{1} \leqslant b_{1}$ for arbitrary $u_{2} \geqslant 0$ we have $g\left(t_{1}, u_{2}\right) \geqslant 0$. Then $G^{(1,0)}\left(t_{1}, t_{2}\right) \geqslant 0$ for arbitrary point $\left(t_{1}, t_{2}\right) \in\left(\left(a_{1}+b_{1}\right) / 2, b_{1}\right] \times\left[a_{2}, b_{2}\right]$. It is obviously, that for arbitrary $u_{2} \geqslant 0$ $g\left(\left(a_{1}+b_{1}\right) / 2, u_{2}\right)=0$. Then, $G^{(1,0)}\left(\left(a_{1}+b_{1}\right) / 2, t_{2}\right)=0$ for arbitrary $t_{2} \in\left[a_{2}, b_{2}\right]$.

By using the similar reasoning we can get, that $G^{(0,1)}\left(t_{1}, t_{2}\right)$ is non-positive on the set $\left[a_{1}, b_{1}\right] \times$ $\left[a_{2},\left(a_{2}+b_{2}\right) / 2\right)$ and nonnegative on the set $\left[a_{1}, b_{1}\right] \times\left(\left(a_{2}+b_{2}\right) / 2, b_{2}\right]$. And $G^{(0,1)}\left(t_{1},\left(a_{2}+b_{2}\right) / 2\right)=0$ for arbitrary $t_{1} \in\left[a_{1}, b_{1}\right]$.

From the above we can conclude that the vertices of the rectangle $\left[a_{1}, b_{1}\right] \times\left[a_{2}, b_{2}\right]$ are the points in which the function $G\left(t_{1}, t_{2}\right)$ takes the maximum value. Substituting the coordinates of the vertices in the formula (8) we can ensure that the equality (7) is hold. The lemma 1 is proved.

Lemma 2. If the function $f\left(t_{1}, t_{2}\right)$ meets the requirements of the lemma 1 then the function

$$
Q\left(t_{1}, t_{2}\right) \stackrel{\mathrm{df}}{=} \frac{1}{t_{1} t_{2}} \int_{0}^{t_{1}} \int_{0}^{t_{2}} f\left(u_{1}, u_{2}\right) \mathrm{d} u_{1} \mathrm{~d} u_{2}
$$

where $0<t_{i}<\infty(i=1,2)$, is nondecreasing for each variables. 
Proof of the lemma 2. Because of the function $f$ is nondecreasing for each variables then

$$
\frac{1}{t_{1}} \int_{0}^{t_{1}} f\left(u_{1}, u_{2}\right) \mathrm{d} u_{1} \leqslant f\left(t_{1}, u_{2}\right)
$$

where $0<u_{2}<\infty$. Using Fubini's theorem and inequality (10) we have

$$
Q^{(1,0)}\left(t_{1}, t_{2}\right)=\frac{1}{t_{1} t_{2}}\left\{\int_{0}^{t_{2}} f\left(t_{1}, u_{2}\right) \mathrm{d} u_{2}-\frac{1}{t_{1}} \int_{0}^{t_{1}} \int_{0}^{t_{2}} f\left(u_{1}, u_{2}\right) \mathrm{d} u_{1} \mathrm{~d} u_{2}\right\} \geqslant 0 .
$$

Analogous we obtain $Q^{(0,1)}\left(t_{1}, t_{2}\right) \geqslant 0$. Hence, in view of (11), we have the statement of the lemma 2.

Proof of the theorem 1. Using the reasoning analogous to the case of the function of one variable $[28,29]$, we can write the $S_{n_{1}, \infty}(f), S_{\infty, n_{2}}(f)$ as follows:

$$
\begin{aligned}
& S_{n_{1}, \infty}\left(f ; t_{1}, t_{2}\right)=\left\|\Lambda_{m_{1}}^{\nu_{1}}\right\|^{-1} \int_{\Lambda_{m_{1}}^{\nu_{1}}} f\left(u_{1}, t_{2}\right) \mathrm{d} u_{1}, \text { if } t_{1} \in \Lambda_{m_{1}}^{\nu_{1}} \forall t_{2} \in \mathbb{I}, \\
& S_{\infty, n_{2}}\left(f ; t_{1}, t_{2}\right)=\left\|\Lambda_{m_{2}}^{\nu_{2}}\right\|^{-1} \int_{\Lambda_{m_{2}}^{\nu_{2}}} f\left(t_{1}, u_{2}\right) \mathrm{d} u_{2}, \text { if } t_{2} \in \Lambda_{m_{2}}^{\nu_{2}} \forall t_{1} \in \mathbb{I},
\end{aligned}
$$

where $\Lambda_{m_{i}}^{\nu_{i}}(i=1,2)$ is setted by the formula (4). In the common points for the segments $\overline{\Lambda_{m_{1}}^{\nu_{1}}}\left(\overline{\Lambda_{m_{2}}^{\nu_{2}}}\right)$, the function $S_{n_{1}, \infty}(f)\left(S_{\infty, n_{2}}(f)\right)$ is equal to the half-sum of the values from the intervals $\Lambda_{m_{1}}^{\nu_{1}}\left(\Lambda_{m_{2}}^{\nu_{2}}\right)$. The function $S_{n_{1}, \infty}\left(f ; t_{1}, t_{2}\right)$ in the points $\left(0, t_{2}\right)$ and $\left(1, t_{2}\right)$ is continuous for arbitrary $t_{2} \in \mathbb{I}$, and the function $S_{\infty, n_{2}}\left(f ; t_{1}, t_{2}\right)$ is continuous in the points $\left(t_{1}, 0\right)$ and $\left(t_{1}, 1\right)$ for arbitrary $t_{1} \in \mathbb{I}$.

From the results of M.N. Ochirov [20] and L.G. Khomutenko [21], based on the standard reasoning from [29](p.21) and [26](p.57-58), we can conclude that for arbitrary $\mathbf{t}=\left(t_{1}, t_{2}\right) \in \Lambda_{m_{1}, m_{2}}^{\nu_{1}, \nu_{2}}$ and $\mathbf{n}=\left(n_{1}, n_{2}\right)\left(n_{i}=2^{m_{i}}+k_{i} ; m_{i} \in \mathbb{Z}_{+} ; k_{i}=\overline{1,2^{m_{i}}} ; i=1,2\right)$ the Fourier-Haar partial rectangle sum of the order $\mathbf{n}$ of the function $f(\mathbf{t}) \in C$ can be written as follows

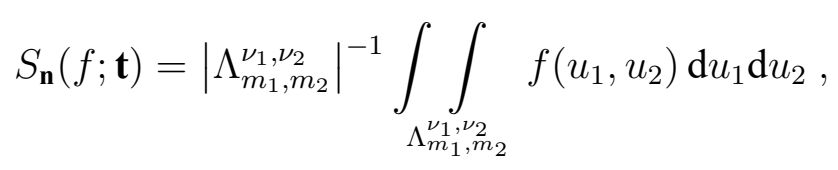

where $\Lambda_{m_{1}, m_{2}}^{\nu_{1}, \nu_{2}}=\Lambda_{m_{1}}^{\nu_{1}} \times \Lambda_{m_{2}}^{\nu_{2}}$. In the points common for the closure of the sets $\Lambda_{m_{1}, m_{2}}^{\nu_{1}, \nu_{2}}$ the function $S_{\mathbf{n}}(f ; \mathbf{t})$ is equal to the average of the values from the rectangles $\Lambda_{m_{1}, m_{2}}^{\nu_{1}, \nu_{2}}$ that abut for these points. Herewith $S_{\mathbf{n}}(f ; \mathbf{t})$ is continuity in the boundary points of the domain $\mathbb{I}^{2}$ that do not match to the points in which the rectangles $\Lambda_{m_{1}, m_{2}}^{\nu_{1}, \nu_{2}}$ abut one to another. 
For arbitrary function $f \in H_{\Omega}$ and for arbitrary $\mathbf{n} \in \mathbb{N}_{*}^{2}$ and $\mathbf{t}=\left(t_{1}, t_{2}\right) \in \Lambda_{m_{1}, m_{2}}^{\nu_{1}, \nu_{2}}$ using (14) and (12)-(13) we have

$$
\begin{aligned}
\left|f(\mathbf{t})-\sigma_{\mathbf{n}}(f ; \mathbf{t})\right| & =\mid f\left(t_{1}, t_{2}\right)-\frac{1}{\left|\Lambda_{m_{1}}^{\nu_{1}}\right|} \int_{\Lambda_{m_{1}}^{\nu_{1}}} f\left(u_{1}, t_{2}\right) \mathrm{d} u_{1}- \\
& -\frac{1}{\left|\Lambda_{m_{2}}^{\nu_{2}}\right|} \int_{\Lambda_{m_{2}}^{\nu_{2}}} f\left(t_{1}, u_{2}\right) \mathrm{d} u_{2}+\frac{1}{\left|\Lambda_{m_{1}, m_{2}}^{\nu_{1}, \nu_{2}}\right|} \iint_{\Lambda_{m_{1}, m_{2}}^{\nu_{1}, \nu_{2}}} f\left(u_{1}, u_{2}\right) \mathrm{d} u_{1} \mathrm{~d} u_{2} \mid .
\end{aligned}
$$

Because of $\Lambda_{m_{1}, m_{2}}^{\nu_{1}, \nu_{2}}=\Lambda_{m_{1}}^{\nu_{1}} \times \Lambda_{m_{2}}^{\nu_{2}}$ and using (4) we have Then from the (15) we obtain

$$
\begin{aligned}
\mid f(\mathbf{t}) & -\sigma_{\mathbf{n}}(f ; \mathbf{t}) \mid \leqslant \\
& \leqslant\left|\Lambda_{m_{1}, m_{2}}^{\nu_{1}, \nu_{2}}\right|^{-1} \iint_{\Lambda_{m_{1}, \nu_{2}}^{\nu_{2}}}\left|f\left(t_{1}, t_{2}\right)-f\left(u_{1}, t_{2}\right)-f\left(t_{1}, u_{2}\right)+f\left(u_{1}, u_{2}\right)\right| \mathrm{d} u_{1} \mathrm{~d} u_{2} \leqslant \\
& \leqslant\left|\Lambda_{m_{1}, m_{2}}^{\nu_{1}, \nu_{2}}\right|^{-1} \iint_{\Lambda_{m_{1}, m_{2}}^{\nu_{1}, \nu_{2}}} \Omega\left(\left|u_{1}-t_{1}\right|,\left|u_{2}-t_{2}\right|\right) \mathrm{d} u_{1} \mathrm{~d} u_{2} .
\end{aligned}
$$

The modulus of continuity $\Omega(\mathbf{u})$ meets all requirements of the lemma 1 . Then for arbitrary point $\mathbf{t} \in \Lambda_{m_{1}, m_{2}}^{\nu_{1}, \nu_{2}}$ from the (16) it follows

$$
\left|f\left(t_{1}, t_{2}\right)-\sigma_{n_{1}, n_{2}}\left(f ; t_{1}, t_{2}\right)\right| \leqslant n_{1}^{\prime} n_{2}^{\prime} \int_{0}^{1 / n_{1}^{\prime}} \int_{0}^{1 / n_{2}^{\prime}} \Omega\left(u_{1}, u_{2}\right) \mathrm{d} u_{1} \mathrm{~d} u_{2} \quad \forall f \in H_{\Omega} .
$$

Let us show that in the points of the junction of the rectangles $\Lambda_{m_{1}, m_{2}}^{\nu_{1} \nu_{2}}$ the inequality (17) is hold. For this purpose we consider the arbitrary function $f \in H_{\Omega}$ and the point $\mathbf{t}^{*}=\left(t_{1}^{*}, t_{2}^{*}\right)$ such that $t_{i}^{*} \stackrel{\mathrm{df}}{=} k_{i} / 2^{m_{i}}\left(k_{i}=\overline{1,2^{m_{i}}-1} ; m_{i} \in \mathbb{N} ; i=1,2\right)$. In the point $\mathbf{t}^{*}$ the four rectangles $\Lambda_{m_{1}, m_{2}}^{\nu_{1}, \nu_{2}}$ are abutted one to another. Applying the lemmas 1 and 2 we obtain

$$
\begin{aligned}
\mid f\left(\mathbf{t}^{*}\right) & -\sigma_{\mathbf{n}}\left(f ; \mathbf{t}^{*}\right) \mid \leqslant 2^{m_{1}+m_{2}-2}\left\{4 \iint_{\triangle_{m_{1}+1, m_{2}+1}} \Omega\left(\left|t_{1}^{*}-u_{1}\right|,\left|t_{2}^{*}-u_{2}\right|\right) \mathrm{d} u_{1} \mathrm{~d} u_{2}+\right. \\
& +2 \iint_{\triangle_{m_{1}+1, m_{2}}^{2 k_{1}, k_{2}+1}} \Omega\left(\left|t_{1}^{*}-u_{1}\right|,\left|t_{2}^{*}-u_{2}\right|\right) \mathrm{d} u_{1} \mathrm{~d} u_{2}+2 \iint_{\triangle_{m_{1}, m_{2}+1}^{k_{1}+1,2 k_{2}}} \Omega\left(\left|t_{1}^{*}-u_{1}\right|,\left|t_{2}^{*}-u_{2}\right|\right) \mathrm{d} u_{1} \mathrm{~d} u_{2}+ \\
& \left.+\iint_{\triangle_{m_{1}, m_{2}}} \Omega\left(\left|t_{1}^{*}-u_{1}\right|,\left|t_{2}^{*}-u_{2}\right|\right) \mathrm{d} u_{1} \mathrm{~d} u_{2}\right\} \leqslant 2^{m_{1}+m_{2}} \int_{0}^{2^{-m_{1}}} \int_{0}^{2^{-m_{2}}} \Omega\left(u_{1}, u_{2}\right) \mathrm{d} u_{1} \mathrm{~d} u_{2}= \\
= & n_{1}^{\prime} n_{2}^{\prime} \int_{0}^{1 / n_{1}^{\prime} 1 / n_{2}^{\prime}} \int_{0} \Omega\left(t_{1}, t_{2}\right) \mathrm{d} t_{1} \mathrm{~d} t_{2} .
\end{aligned}
$$


Analogous we can prove that (17) is hold in all other junction points of the rectangles $\Lambda_{m_{1}, m_{2}}^{\nu_{1}, \nu_{2}}$. From the above and (17) we have

$$
\sup _{f \in H_{\Omega}}\left\|f-\sigma_{n_{1}, n_{2}}(f)\right\|_{C} \leqslant n_{1}^{\prime} n_{2}^{\prime} \int_{0}^{1 / n_{1}^{\prime}} \int_{0}^{1 / n_{2}^{\prime}} \Omega\left(t_{1}, t_{2}\right) \mathrm{d} t_{1} \mathrm{~d} t_{2} .
$$

Let us consider the function $\mathcal{I}\left(t_{1}, t_{2}\right) \stackrel{\mathrm{df}}{=} \Omega\left(1-t_{1}, 1-t_{2}\right)$. Using the properties of the semiadditivity and nondecreasing on each variables of the modulus of continuity $\Omega$, we can show that $\mathcal{I} \in H_{\Omega}$. Then we obtain the lower bound

$$
\begin{aligned}
\sup _{f \in H_{\Omega}}\left\|f-\sigma_{n_{1}, n_{2}}(f)\right\|_{C} & \geqslant\left\|\mathcal{I}-\sigma_{n_{1}, n_{2}}(\mathcal{I})\right\|_{C} \geqslant\left|\mathcal{I}(1,1)-\sigma_{n_{1}, n_{2}}(\mathcal{I} ; 1,1)\right|= \\
& =\left|\sigma_{n_{1}, n_{2}}(\mathcal{I} ; 1,1)\right|=n_{1}^{\prime} n_{2}^{\prime} \int_{0}^{1 / n_{1}^{\prime}} \int_{0}^{1 / n_{2}^{\prime}} \Omega\left(t_{1}, t_{2}\right) \mathrm{d} t_{1} \mathrm{~d} t_{2} .
\end{aligned}
$$

The equality (6) for arbitrary $\mathbf{n} \in \mathbb{N}_{*}^{2}$ is followed from the estimates (18) and (19). Theorem 1 is proved.

\section{Conclusions}

The exact value of the upper bound of the approximation error by the construction of the type "angle" $\sigma_{n_{1}, n_{2}}\left(f ; t_{1}, t_{2}\right)$ built using the Fourier-Haar partial sums is obtained in the uniform metric on the function class of two variables defined using of the mixed modulus of continuity $\Omega\left(t_{1}, t_{2}\right)$.

\section{References}

[1] A. Haar, Zur Theorie der orthogonalen Funktionensysteme. Inaugural-dissertation, GeorgAugust-Universitat, Göttingen, 1909.

[2] P.L. Ulyanov, Series in Haar system, Matem. sbornik. 63:1 (1964) 356-391. (in Russian)

[3] Z. Ciesielski, On Haar functions and on the Schauder Basis of the Space $C_{\langle 0,1\rangle}$, Bulletin de L'academie Polonaise Des Sciences. Serie des sci. math., astr. et phys. 7:4 (1959) 227-232.

[4] B.I. Golubov, On Fourier series of continuous functions with respect to a Haar system, Izv. Akad. Nauk SSSR Ser. Mat. 28 (1964) 1271-1296. (in Russian)

[5] I. I. Sharapudinov, Approximation of functions in variable-exponent Lebesgue and Sobolev spaces by finite Fourier-Haar series, Sb. Math. 205:2 (2014) 291-306.

[6] S.Yu. Galkina, On the Fourier-Haar coefficients of functions of bounded variation, Mathematical Notes. 51:1 (1992) 27-36.

[7] S.Yu. Galkina, On the Fourier-Haar Coefficients of Functions of Several Variables with Bounded Vitali Variation, Mathematical Notes. 70:5 (2001) 733-743.

[8] S.S. Volosivets, Approximation of functions of bounded p-variation by means of polynomials of the Haar and Walsh systems, Mathematical Notes. 53:6 (1993) 569-575.

[9] A.N. Shchitov, Sharp estimates of the Fourier-Haar coefficients of some classes of functions of several variables, Zb. Pr. Inst. Mat. NAN Ukr. 1:1 (2004) 413-427. (in Russian)

[10] A.N. Shchitov, The Exact Estimates of Fourier-Haar Coefficients of Functions of Bounded Variation, International Journal of Advanced Research in Mathematics. 4 (2016) 14-22. 
[11] M.G. Plotnikov, Coefficients of convergent multiple Haar series, Russian Mathematics. 56:1 (2012) 61-65.

[12] B.I. Golubov, Series with respect to the Haar system, J. Soviet Math. 1:6 (1973) 704-726.

[13] N.P. Khoroshko, On the best approximation in the metric of $L$ to certain classes of functions by Haar-system polynomials, Mathematical notes of the Academy of Sciences of the USSR. 6:1 (1969) 487-491.

[14] N.P. Khoroshko, Uniform approximation in classes of continuous functions by polynomials in the Haar system, Ukrainian Math. J. 22:5 (1971) 611-618. (in Russian)

[15] S.B. Vakarchuk, A.N. Shchitov, On the best approximation of functions of bounded p-variation by Haar polynomials, Bulletin of the Dnipropetrovsk University. Mathematics. 11 (2004) 28-34. (in Russian)

[16] S.B. Vakarchuk, A.N. Shchitov, Estimates of the best one-sided approximation of some classes of functions by polynomials constructed on Haar system, Zb. Pr. Inst. Mat. NAN Ukr. 4 (2007) 7-22. (in Russian)

[17] S.B. Vakarchuk, A.N. Shchitov, Estimates for the error of approximation of functions in $L_{p}^{1}$ by polynomials and partial sums of series in the Haar and Faber-Schauder systems, Izvestiya Mathematics. 79:2 (2015) 257-287.

[18] A.R. Abdulgamidov, On some properties of the Fourier-Haar series of the functions of two variables, Proceedings of the postraduate papers of the Kazan state university: Mathematics, Mechanics, Physics. (1968) 5-33. (in Russian)

[19] N.D. Rishchenko, On the best approximation of the function of the several variables by the step functions, Proccedings of the Dnipropetrovsk state university. (1969) 56-59. (in Russian)

[20] M.N. Ochirov, On approximation of functions of two variables by Fourier-Haar partial sums, Proccedings of the Kazan state university: Function analyse and function theory. 8 (1971) 142145. (in Russian)

[21] L.G. Khomutenko, Uniform approximation of functions of two variables by polynomials in the Haar system, Proccedings of the Dnipropetrovsk state university. (1973) 85-87. (in Russian)

[22] P.V. Zaderey, N.N. Zaderey, Uniform approximations by polynomials in the Haar system on classes of continuous functions of several variables, Zb. Pr. Inst. Mat. NAN USSR. (1984) 6066. (in Russian)

[23] S.B. Vakarchuk, A.N. Shchitov, Uniform approximation of some classes of functions of several variables by polynomials constructed by Haar system and partial sums of the Fourier-Haar series, Zb. Pr. Inst. Mat. NAN Ukr. 1:1 (2004) 42-59. (in Russian)

[24] M.K. Potapov, The study of some classes of functions by the help of the approximation by the "angle", Proceedings of the Steklov Institute of Mathematics. 117 (1972) 256-291. (in Russian)

[25] M.K. Potapov, On the approximation by the "angle", Proceedings of the Conference of Constructive Theory of Functions. 1969. (in Russian)

[26] G. Alexits, Convergence problems of orthogonal series, Akademia Kiado, Budapest, Hungary, 1961.

[27] B. S. Kashin, A. A. Saakyan, Orthogonal series, Transl. Math. Monogr., 75, Amer. Math. Soc., Providence, RI, 1989. 
[28] S.S. Kaczmarz, H. Steinhaus, Theorie der Orthogonalreihen, Monografje Matematyczne, VI, Chelsea Publishing Company, New York, 1951.

[29] I.M. Sobol, Multidimensional quadrature formulas and functions Haar, Nauka, Moscow, USSR, 1969.

[30] O.D.Gabisonia, On the value of the best approximation of functions of several variables by polynomials in the Haar system, Izv. Vyssh. Uchebn. Zaved., Mat. 1 (1968) 39-46. (in Rusian) 